\title{
Detection of temporal gaps within and between perceptual tonal groups*
}

\author{
PETER J. FITZGIBBONS and ALEXANDER POLLATSEK \\ Department of Psychology \\ and \\ IAN B. THOMAS \\ Electrical and Computer Engineering Department \\ University of Massachusetts, Amherst, Massachusetts 01002
}

\begin{abstract}
Two experiments were conducted to explore the hypothesis that tones widely separated in frequency are processed in separate communication channels. Listeners attempted to detect brief temporal gaps between items in a simple tonal sequence of two high tones followed by two low tones. Temporal resolution within both the high and low groups was near perfect; between-group detection scores were significantly lower. Results are interpreted as evidence for a processing time delay when shifts of focal attention occur between perceptual structures organized within the frequency domain.
\end{abstract}

If a temporal gap is placed between two easily detected auditory inputs, is its detectability simply a function of the physical duration of the gap? One hypothesis that suggests that it is not is attention switching.

If the input before the gap were encoded in one communication channel (A) and the input after the gap were encoded in a different communication channel $(B)$, and if the mechanism to switch attention from Channel A to Channel B took longer than the duration of the gap, then the gap might go undetected. More precisely, if the listener is attending to Channel $A$ and if the offset of the tone in that channel is the signal for him to switch attention to Channel B, then Channel B will already have a signal present by the time the listener's central processor gets there, if the switch time is greater than the gap duration. Thus, assuming that no stimulus information gets into the central processor during switching, this stimulus situation should be indistinguishable from one in which no gap is present. Thus, the perceptibility of a physical gap should depend on whether the stimuli preceding and following the gap are in the same channel or not.

The present experiments were conducted to explore the hypothesis that attention switching influences the ability of listeners to detect gaps. Since the experiments attempted to create two information channels by varying frequency, experiments in which

\footnotetext{
*This research was supported by Grant NS-08306 from the National Institutes of Health to I. B. Thomas, and is based on a Master's thesis submitted to the University of Massachusetts by P. J. Fitzgibbons.

†Requests for reprints should be sent to Ian B. Thomas, Dynastat, Inc., 2704 Rio Grande, Austin, Texas 78705.
}

large differences in pitch seem to create separate information channels will be briefly reviewed.

Bregman and Campbell (1971) discussed references to a perceptual phenomenon which they called primary auditory stream segregation (PASS). The term was used in reference to a listening experience in which a single sequence of rapidly occurring auditory events seem to "split," perceptually, into subsets of related sounds as though each distinctive group was cooccurring in time with other such groups. To observe the effect of PASS on listeners' temporal ordering performance, Bregman and Campbell presented a repetitive sequence of six sine tones, three from a high-frequency range and three from a lower frequency category. Each tone was $100 \mathrm{msec}$ in duration, with discrete frequency transitions between temporally contiguous stimuli. At that presentation rate $(10$ tones $/ \mathrm{sec})$, the grouping of stimuli by frequency category was subjectively experienced by all the listeners. Analysis of ordering performance revealed that listeners were readily able to order correctly the tones within either the high- or low-frequency streams, but were incapable of relating the temporal positions of tones belonging to different frequency categories.

Similar perceptual organizations have been observed to inhibit, or facilitate, the sequential processing of various auditory patterns. Warren, Obusek, Farmer, and Warren (1969) employed the repetitive sequencing paradigm and reported that naive listeners displayed no ability to correctly order four qualitatively different sounds (sine tone, hiss, vowel, and buzz), each $200 \mathrm{msec}$ in duration. Though each sound in the sequence was identified by the listeners, they reported no better than chance performance in identifying the sequential nature of 
the stimuli. Bregman and Campbell hypothesized that the confusion of listeners resulted from the development of multiple auditory streams which essentially destroyed the perceptual continuity of the sequence.

Warren et al also reported that when the unrelated sounds of the sequence were replaced by four spoken digit stimuli, listeners demonstrated no confusion in reporting the correct order of sounds at the same presentation rate $(200 \mathrm{msec} / \mathrm{item})$. This finding was supported by results from a study by Thomas, Hill, Carroll, and Bienvenida (1970) which reported that listeners were able to report the correct order for a repeating sequence of four natural vowel sounds when segment durations exceeded $125 \mathrm{msec}$. Perceptual grouping and chance level performances were observed at faster presentation rates. Similar "speech-like" performance functions were subsequently obtained by employing sequences of all high tones (e.g., 1,976, 1,568, 2,093, and $1,760 \mathrm{~Hz}$ ) or all low tones $(587,659,523$, and $740 \mathrm{~Hz})$, presented in a repetitive manner (Thomas \& Fitzgibbons, 1971). However, if the tones of a sequence alternated between high and low frequencies, segment durations had to be greater than $300 \mathrm{msec}$ before listeners reported correct temporal orders. While these results may not reflect absolute "thresholds" for ordering performance, they do suggest that the temporal course of information processing, as well as the perceptual organization, may be influenced by physical parameters of the stimulus pattern.

Of particular interest to the present discussion of auditory streams is the compelling degree of phenomenal separation between perceptual groups under conditions of PASS. On the basis of this, Bregman and Campbell suggested that the time-limiting process in perceiving accurate sequential order was the necessary shifting of attention from stream to stream. In that sense, the segregated streams would seem to possess properties similar to the physically isolated messages of dichotic listening studies, some of which report that listeners can attend to only one ear channel at a time. Neisser (1967) and Moray (1969) reviewed many of the studies dealing with that issue.

Aside from certain procedural differences between the dichotic listening paradigm and that of the ordering studies cited above, there seems to be at least one important difference which reflects on the degree of perceptual "distance" between auditory streams. Though certain studies report that the ears may function as independent sensory channels (Treisman, 1971), no conclusive evidence has been offered which suggests that they must function in that manner. In fact, several studies have reported an inability on the part of listeners to selectively ignore information sent to a specified ear channel (Day, 1968; Treisman, 1970). Such findings suggest that, for certain testing conditions, the two ears function as a single sensory channel, with listeners processing a fusion of dichotic inputs. Listeners experiencing the perceptual grouping characteristic of PASS demonstrate no similar ability to fuse stimulus information across streams, even when task demands require such an operation.

The two following experiments were conducted in an attempt to explore further the assumption that a central switching process is operative during the sequential tracking of tonal stimuli. If frequency manipulations can produce predictable effects on the perceptibility of gaps, then one would have a second converging operation (in addition to temporal order judgments) to the perceptual quality of distal separation between segregated streams.

\section{EXPERIMENT I}

This experiment investigated the effect of discrete frequency transitions on the detection of temporal interruptions in a rapid tonal pattern. It was thought that one result of a time-dependent attention switch, assumed to occur between frequency categories, would be a momentary interruption in the processing of information. As outlined above, the attentionswitching hypothesis states that listeners should experience extreme difficulty in detecting brief temporal gaps in a tonal sequence when the gaps occurred between tones of different frequency groups. On the other hand, since tones in a narrow frequency range were presumed to be processed as "single-channel" events, temporal interruptions within such pattern locations were expected to be readily detected.

\section{Method}

Tonal Sequences. The primary auditory pattern comprised two sine tones from a high- $(\mathrm{H})$ frequency range $(2,093$ and $2,394 \mathrm{~Hz}$ ), and two from a range three octaves lower (L) in frequency (440 and $494 \mathrm{~Hz}$ ). The tones were ordered 2,093, 2,394, 440, and $494 \mathrm{~Hz}$ $\left(\mathrm{H} \mathrm{H} \mathrm{L} \mathrm{L)}\right.$, with corresponding musical notation $\mathrm{C}_{7} \mathrm{D}_{7} \mathrm{~A}_{4} \mathrm{~B}_{4}$ ). The tones were physically grouped by frequency domain to avoid the perceptual cooccurrence of tonal streams, characteristic of the PASS phenomenon.

Long samples of each frequency were recorded using a high-quality signal generator (HP 650A) and tape recorder (General Radio 1525). Care was taken to insure constancy of frequency (GR 1151-A digital counter) and intensity (true RMS meter, HP 3400A), for each recorded sample. Sequences were constructed by splicing tape segments of appropriate length (and thus, duration). All segments had a steady-state duration of $150 \mathrm{msec}$ with $45-\mathrm{deg}$ splicing angles, so that the offset amplitude decay of each tone was coincident with the amplitude buildup of the succeeding tone. Duration of tonal overlap was observed on oscilloscope to be approximately $5 \mathrm{msec}$; thus, total item durations were $160 \mathrm{msec}$. Angular splicing was employed to avoid perceptual clicks associated with instantaneous transitions to "silent" intervals.

Nine comparison sequences were constructed by inserting each of three gap (G) tape segments $(20,40,80 \mathrm{msec})$ into one of three possible locations within the HHLL sequence: HGHLL, HHGLL, or HHLGL. Each comparison sequence was, therefore, greater in 
overall duration than a standard sequence for which all tones were temporally contiguous. Another nine comparison patterns employed the same sine tones but reversed in sequential order, LLHH. A control sequence of four low-frequency segments (LLLL) was employed with frequencies ordered $466,494,415$, and $440 \mathrm{~Hz}$. The tones selected were all within a range of three semitones $\left(A_{4}^{\#}, B_{4}\right.$, $G_{4}^{\#}$, and $A_{2}$ ) to insure their inclusion into a unitary perceptual organization. As an added control measure, the frequency arrangement of the control sequence was ordinally the same as the HHLL sequence. Six comparison tapes contained the control sequence, each with a 20 -msec gap inserted in one of the three locations with two sequence orders.

Thus, 24 comparison sequences were constructed: 18 for the HHLL pattern ( 3 gaps by 3 locations by 2 sequence orders), and 6 for the control pattern ( 1 gap by 3 locations by 2 sequence orders). Practice tapes were also constructed to correspond to the comparison sequences, but employed 200-msec gaps.

Each tape was subsequently formed into a physical loop with a standard sequence on one track of the tape and a comparison sequence on the other track. The standard and comparison sequences on each tape were the same, with the exception that the comparison sequence contained a single gap in one location.

Testing Procedure. Six undergraduate students from the Psychology Department at the University of Massachusetts participated as listeners in the experiment. All students were individually tested and paid for their services. Twelve experimental conditions were conducted with each listener, four tapes in a given session on 3 consecutive days. Within each session, three of the nine HHLL and one of the LLLL patterns were presented, with half the listeners receiving one sequence order and the other three listeners receiving the reversed order. Each sequence presented within a session contained a temporal gap of different duration inserted at a different location. Each listener received the 12 conditions in a different order, and the gap duration presentation order was counterbalanced across both listeners and testing sessions. Listening was done binaurally in phase through headphones (Koss PRO-4) at $80 \mathrm{~dB}$ SPL (re: .0002 dynes $/ \mathrm{cm}^{2}$ ). At that intensity level, the tones of the sequence were judged to be approximately equal in perceived loudness.

Fifty trials were conducted for each of the 12 conditions with each listener. A single trial consisted of the presentation of either a standard or comparison sequence, twice, with a 1.2-sec intersequence interval. The listeners were requested to report verbally "yes" or "no" with regard to whether or not the twice-presented sequence contained the temporal gap in a previously indicated location. Ss were encouraged to guess when uncertain. Each block of 50 trials employed a single standard and comparison sequence, with a single gap placed in the same location. Before each block of trials, listeners were presented with the appropriate practice tape, with a $200-\mathrm{msec}$ gap inserted at the same sequence location as would subsequently be employed throughout the next 50 trials. Practice trials were conducted until the listener became familiar with the task and sequence type, as evidenced by their meeting a criterion of 10 consecutive correct responses. After each practice session, the listener was again instructed about which sequence location might contain the temporal gap, and also informed of a $50 \%$ probability of occurrence for trials containing the gap. Trial-by-trial feedback was also given to further prevent nonperceptual factors from entering into listener decisions. Twenty-five gap trials were randomly distributed within each experimental block.

\section{Results}

A score for each $\mathrm{S}$ was calculated to designate the proportion (P) of correct responses for each experimental block of trials. Preliminary analysis of data revealed no significant effect of presentation order (HHLL vs LLHH), which suggested that direction of frequency shift did not affect detection

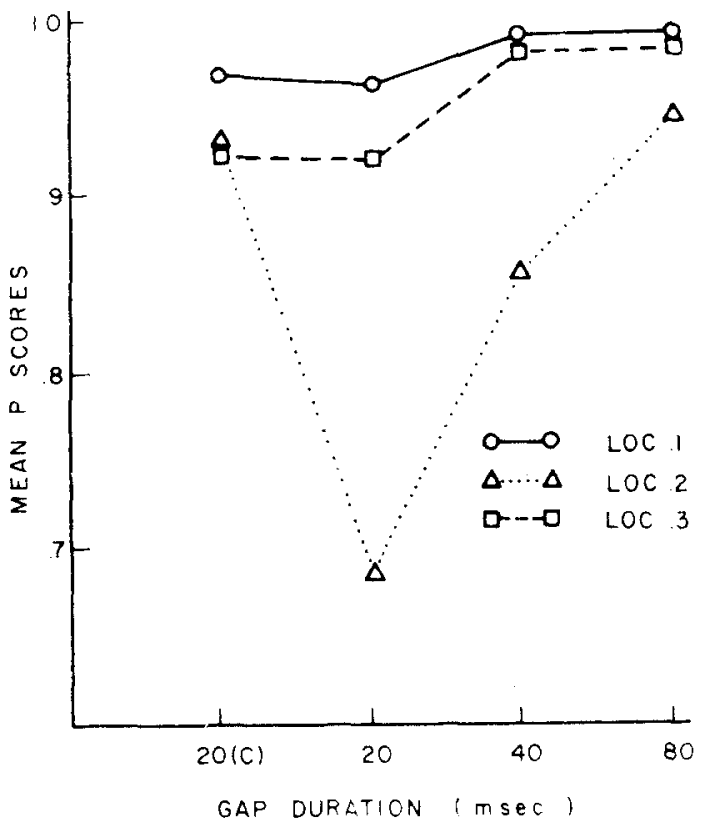

Fig. 1. Mean proportion correct as a function gap duration for each sequence location.

performance, $F(1,4)=1.01, p>.05$. Subsequent analysis was performed on data collapsed across listener groups with gap locations referred to the HHLL sequence order. Figure 1 displays the mean $\mathbf{P}$ scores of listeners for each sequence location as a function of temporal gap durations. Scores for the $20(\mathrm{C})$-msec gap duration refer to locations of the LLLL control sequence. The other scores for Locations 1, 2, and 3 correspond, respectively, to ConditionsHGHLL, HHGLL, and HHLGL.

Analysis of variance of $\mathrm{P}$ scores revealed significant main effects for both gap location, $F(2,10)=17.2$, $p<.001$, and gap duration, $F(3,15)=16.35$, $\mathrm{p}<.001$. A high level of performance (greater than $90 \%$ ) was observed for detection of temporal gaps in Locations 1 and 3 of both sequence types. No significant difference between scores was observed for those locations, which corresponded to interruptions within frequency groups, $\mathrm{F}(1,10)=3.18, \mathrm{p}<.05$. The interaction effect between gap location and gap duration was also found to be significant, $F(6,30)=$ $3.06, p<.025$, which was an expected outcome of the attention-switching hypothesis. A final analysis of mean contrasts revealed that the significantly lower performance levels for Gap Location 2 conditions (than average for other locations) was characteristic solely of the HHLL sequence, $F(1,15)=12, p<.005$. Listeners displayed no detection difficulties for gaps located in the middle position of the control sequence.

In order to rule out criterion shifting explanations of the data, the "hits" (probability of detecting a gap when one was present) and "correct rejections" 
(probability of detecting no gap when one was absent) were tabulated separately in Table 1 . There is almost a perfect correlation between the two since the baseline probability of responding that a gap was present was about .50 for all conditions. For this reason, and since many of the probabilities are close to one, a formal signal detection analysis was not performed.

The results demonstrate that, with the exception of middle location gaps of the HHLL pattern, the listeners' detection performance was quite good for all durations of the inserted gap. The 20- and 40-msec gaps at the frequency-shift location of the HHLL tonal sequence presented more difficulty to listeners than did any of the other experimental conditions. These findings support the hypothesis that listeners' attempts to sequentially process rapid transitions between frequency categories effected temporal discontinuity in a physically continuous pattern.

\section{EXPERIMENT II}

Generally stated, the required task for listeners in Experiment I was to detect any consistent perceptual differences between patterns within a trial block. Through trial-by-trial feedback, such differential cues could have formed the basis of discrimination learning, eventually resulting in correct listener responses. On some level, the most noticeable gap effect could be described as a difference in tonal rhythm between the standard and comparison patterns. Such rhythmic variations were reported often by listeners when referring to conditions of temporal gaps inserted at sequence locations marked by little frequency disparity. Apparently, the small between-group gaps (e.g., HHGLL) produced less discriminative changes in overall pattern perception. A second experiment was conducted to investigate whether such higher order cues played a significant role in detection performance. Accordingly, small duration variations of those tones bordering the inserted gap were made to try to manipulate tonal rhythm. The HHGLL condition with a 20-msec gap was chosen for Experiment II, since the relative contribution of cues other than true "gap detection" would be maximized.

The simple attention-switching hypothesis for the observed performance in Experiment $I$ is that detection decisions for the HHGLL pattern were affected by cues emanating from the critical offset-onset interval that separated the respective $\mathrm{H}$ and $\mathrm{L}$ tonal groups. Presumably, the Ss use the perceived offset of the $\mathrm{H}$ tone group as a signal to switch channels and attempt to detect differential $\mathrm{L}$ tone onset cues in order to distinguish standard and comparison patterns within a trial block. According to this model, above-chance performance in the most difficult condition of Experiment I occurred because
Table 1

Mean Values for Proportion of "Hits" (H) and "Correct Rejections" (CR) in the Conditions of Experiment I

\begin{tabular}{|c|c|c|c|c|}
\hline \multirow{2}{*}{$\begin{array}{c}\text { Gap } \\
\text { Duration } \\
\text { (msec) }\end{array}$} & & \multicolumn{3}{|c|}{ Gap Location } \\
\hline & & HGHLL & HHGLL & HHLGL \\
\hline 20 & $\begin{array}{l}\mathrm{H} \\
\mathrm{CR}\end{array}$ & $\begin{array}{l}.96 \\
.94\end{array}$ & $\begin{array}{l}.66 \\
.68\end{array}$ & $\begin{array}{l}.91 \\
.91\end{array}$ \\
\hline 40 & $\begin{array}{l}\text { H } \\
\text { CR }\end{array}$ & $\begin{array}{l}1.00 \\
1.00\end{array}$ & $\begin{array}{l}.83 \\
.85\end{array}$ & $\begin{array}{r}.99 \\
1.00\end{array}$ \\
\hline 80 & $\begin{array}{l}\mathbf{H} \\
\mathrm{CR}\end{array}$ & $\begin{array}{l}1.00 \\
1.00\end{array}$ & $\begin{array}{l}.97 \\
.92\end{array}$ & $\begin{array}{l}.99 \\
.99\end{array}$ \\
\hline $20(\mathrm{C})$ & $\begin{array}{l}\mathrm{H} \\
\mathrm{CR}\end{array}$ & $\begin{array}{l}.95 \\
.96\end{array}$ & $\begin{array}{l}.93 \\
.89\end{array}$ & $\begin{array}{l}.89 \\
.93 \\
\end{array}$ \\
\hline
\end{tabular}

the time to switch attention is not always greater than the gap duration $(20 \mathrm{msec})$. More importantly, this model predicts that small duration modifications on tones bordering the inserted gap would not affect detection performance, so long as the within-gap perceptual cues were not altered.

However, above-chance performance for this condition is possible, even if a 20 -msec gap interval were not sufficiently long to permit differentiation of L-tone onset cues between HHLL and HHGLL patterns. Even if they were lacking such within-gap cues, listeners could have based detection decisions on a delayed perceptual offset of the first $L$ tone following the inserted gap. That is, the L-tone onset information was lost to perception (or heavily attenuated) during the switch of focal attention between frequency groups. However, since the $\mathrm{L}$ tones of standard and comparison sequences were equal in duration, it was expected that a greater part of that tone $\left(\mathrm{L}_{1}\right)$ would be available to perception during comparison trials. Thus, an alternative explanation of above-chance performance in the HHGLL condition ( $\mathrm{G}=20 \mathrm{msec}$ ) is that responses were guided by cues contained in the perceived $\mathrm{H}_{2}$-tone-offset/ $\mathrm{L}_{1}$-toneoffset interval and not the perceived $\mathrm{H}_{2}$-offset $/ \mathrm{L}_{1}$ onset interval. Hence, a small duration decrease of the $\mathrm{H}_{2}$ tone preceding the temporal gap should have little influence on detection performance, whereas a similar decrease of the $\mathrm{L}_{1}$ tone duration following the gap should minimize differences in $\mathrm{H}_{2}$-tone-offset $/ \mathrm{L}_{1^{-}}$ tone-offset interval between the standard and comparison patterns and make gap detection more difficult.

\section{Method}

Tonal Sequence and Design. The single tonal sequence, HHLL, served as the standard auditory pattern throughout the experiment. As constructed in Experiment I, all tonal segments were temporally contiguous and $160 \mathrm{msec}$ in duration. Three experimental tape loops employed the standard sequence, each with a different comparison pattern on one track of the tape. The comparison patterns comprised the same tones and presentation order as the standard, but each was prepared with a 20 -msec temporal gap inserted between the $\mathrm{H}$ and $\mathrm{L}$ tonal groups. Each comparison 
pattern differed only with respect to the duration of a single tonal segment. One condition employed a $20-\mathrm{msec}$ truncation of the $\mathrm{H}$ tone preceding the inserted gap $\left(\mathrm{HH}_{\mathrm{t}} \mathrm{GLL}\right)$, another with the truncation occurring on the $\mathrm{L}$ tone following the gap $\left(\mathrm{HHGL}_{\mathrm{t}} \mathrm{L}\right)$. Both the patterns, then, were equal in overall duration to the standard sequence. The third condition left unchanged all tonal durations of the HHGLL pattern employed in Experiment I.

Nine listeners who had not participated in the first experiment were employed as Ss. The same testing procedure of Experiment I was used in this investigation. Each listener was presented with a block of 50 gap detection trials for each of the three gap conditions. Besides receiving a practice session prior to each trial block, listeners were given additional practice with the various gap sequences employed in Experiment I. Order of trial blocks was counterbalanced across listeners, and each listener received all three gap conditions in a single 1-h session, with 5-min rest periods between blocks. Ss were not informed of the duration modifications which formed the basis of experimental conditions.

\section{Results}

Mean proportion correct (P) scores for each experimental condition are listed in Table 2. Performance variability across listeners was more pronounced than in Experiment $I, F(8,16)=3.54$, $p<.025$, but not as marked as the significant differences across gap conditions, $F(2,16)=13.6$, $p<.001$. The small $p$ score differential between the two "modified" conditions, $\mathrm{HH}_{\mathrm{t}} \mathrm{GLL}$ and HHHGL $\mathrm{H}$, did not represent a significant variance. However, performance on the unmodified condition, HHGLL, was found to represent a significant increase over the average of the two, $F(1,16)=27.1, p<.001$. Comparison of present findings for the HHGLL condition to its respective condition in Experiment I reveals the similar difficulties for both listener groups to detect a brief gap between divergent frequency categories. Again, there were no significant "bias" problems as the "hits" and "correct rejections" were highly correlated (see Table 2).

The results do not completely support all assumptions of either gap detection strategy outlined above. The first hypothesis predicted that detection scores would vary little across the three gap conditions. The responses of only one listener approximated that expectation. All other listeners, however, performed significantly better on the unmodified sequence HHGLL than in the $\mathrm{HHGL}_{\mathrm{t}} \mathrm{L}$ condition. This result was the predicted outcome of the alternative hypothesis, which assumed that deletion of L-tone durational cues would adversely affect detection performance. Within the framework of the two hypotheses, the unexpected $\mathrm{HH}_{\mathrm{t}} \mathrm{GLL}$ scores make sense only if the assumption is made that the pregap truncation did not significantly change the $\mathrm{H}_{2}$ tone's perceived offset (relative to the HHLL sequence) and thus did not change the time of the switch of attention.

This could have happened if something delayed the perceived offset of the $\mathrm{H}_{t}$ tone significantly beyond its actual physical offset. That such a delay could have occurred is supported by two aspects of auditory
Table 2

Mean Proportion Correct (P) With "Hit" (H) and "Correct Rejection" (CR) Data for the Conditions of Experiment II

\begin{tabular}{llccc}
\hline \multirow{2}{*}{$\begin{array}{c}\text { Gap } \\
\text { Duration } \\
\text { (msec) }\end{array}$} & & \multicolumn{3}{c}{ Sequence Condition } \\
\cline { 2 - 5 } & $\mathrm{HH} \mathrm{H}_{\mathrm{t}} \mathrm{GLL}$ & $\mathrm{HHGL}_{\mathrm{t}} \mathrm{L}$ & HHGLL \\
\hline \multirow{2}{*}{20} & $\mathrm{P}$ & .570 & .560 & .695 \\
& $\mathrm{H}$ & .55 & .55 & .69 \\
& $\mathrm{CR}$ & .59 & .57 & .70 \\
\hline
\end{tabular}

stimulation: (a) the relation between sensation growth (loudness) and stimulus duration over an approximate 200-msec energy summation interval (Békésy, 1960; Munson, 1947), and (b) the observation that the decay of auditory sensation proceeds at a slower rate initially following physical offset of stimuli less developed in loudness (Plomp, 1964). Thus, if perceived offset of any auditory stimulus depends upon a decay in sensation to some criterion level, then these two hypotheses together suggest that it is not unreasonable that the time of perceived offset of the $\mathrm{H}_{t}$ tone was delayed. A tentative explanation is thereby provided for the surprising finding that the $\mathrm{HH}_{\mathrm{t}} \mathrm{GLL}$ sequence is very difficult to distinguish from the control HHLL pattern.

\section{DISCUSSION}

The main results from the two experiments were quite striking. Detection of gaps between tones of similar frequency was markedly superior to detection of gaps between tones of dissimilar frequency. The inability of listeners to discriminate HHLL and HHGLL patterns is consistent with the hypothesis that wide discrete frequency discontinuities are also perceived as temporal discontinuities in the flow of information. These results seem best explained in terms of attention shifting within the frequency domain.

\section{Peripheral Interaction Explanations}

One alternative explanation for the results of Experiment $I$ is that some sort of peripheral interaction could have produced a cue indicating whether a gap had occurred or not. In an experiment similar to Experiment I, Collyer (1974) found that gap detection was poorest between two successive stimuli in different modalities (a tone and a light), intermediate between two tones of widely differing frequencies, and easiest between two tones of similar frequencies. He attempted to fit a particular quantitative model of duration discrimination to all three sets of data and found that the model fit the bimodality condition better than the two intramodality conditions. Collyer interpreted this finding as support for the hypothesis that intramodal stimuli are not processed independently, with peripheral cues probably aiding gap detection and, therefore, not the 
preferred stimuli for investigation of central timing mechanisms. Such conclusions, however, were not based on tested quantitative assumptions in the model. Moreover, as Collyer points out, an attention switching model could equally well explain the difference between the "widely spaced" tones and bimodal conditions by assuming that the switching time was greater between modalities than between frequency domains of the auditory modality.

While the present experiments do not provide any critical test of the peripheral interaction hypothesis, it seems less attractive than the attention-switching hypothesis on the basis of available data. However, no claim is made that auditory cues (perhaps peripheral) are never effective as an aid to gap detection in certain stimulus conditions. For example, if stimuli bounding temporal gaps are nearly identical (or processed within the same perceptual channel), it seems conceivable that sensory "on/off" cues or relative decays in sensation level may be discriminated to decide on gap occurrence. Moreover, Abel (1972) has reported that the strength of these cues is somewhat dependent on the physical parameters of the stimuli (amplitude and duration) and their effectiveness a function of the gap durations involved. Accordingly, it seems plausible that auditory cues could have been employed by listeners in discriminating LLLL and LLGLL sequences.

Peripheral interaction explanations seem less powerful when directed at the frequency disparity effect on gap detection. First of all, any peripheral model that relies heavily on the concept of auditory masking seems implausible, since the most effective temporal masking is reported for tones close in frequency (Homick, Elfner, \& Bothe, 1969; Elliott, 1962). Thus, greater confusion would be expected with trial blocks employing the LLLL pattern. Also, little perceptual masking would be expected with the intensity levels in the present experiments. Unless stimuli in different frequency domains are presumed to be processed independently, it is difficult to explain why temporal gaps are perceived in both the HHLL and HHGLL tonal patterns.

Another explanation of gap detection performance in the present experiments would be in terms of discrimination between the total durations of the standard and comparison sequences. However, the discrimination seems implausible for the durations involved, and furthermore, it would not explain the differences observed between the HHLL and LLLL sequences.

\section{Frequency Domains as Communication Channels}

There are some data from experiments using pairs of tones that seem to be at variance with the hypothesis that tones with large frequency separations define separate perceptual channels. If the task is to resolve order relations between two auditory events with onset asynchronies, listener performance is invariably reported as being unaffected by tonal frequency separation (Hirsh, 1957; Norman, 1967). However, studies (in addition to the Collyer, 1974, experiment discussed) that have employed gap detection paradigms report that frequency environment can greatly influence detection scores.

Williams and Perrott (1972) obtained temporal acuity measures for gaps between pulsed tonal pairs, with stimulus duration and frequency separation as experimental variables. For brief tonal durations $(3-30 \mathrm{msec})$, gap thresholds were fairly constant $(3-5 \mathrm{msec})$ and independent of frequency differences. With more extended tones (100-300 msec), thresholds were similarly small and constant, for little frequency disparity, but increased almost linearly as the frequency disparity increased. When broadband "noise" stimuli are employed, gap thresholds are also small (3-7 msec) (Plomp, 1964; Elfner \& Caskey, 1965). An extremely complex peripheral interaction model would seem necessary to explain the marked effects on gap detection caused by the interaction of stimulus duration and frequency. However, these findings may simply indicate that the auditory system requires a certain period to "tune" sensory frequency domains, which, upon development, function as separate perceptual channels. Short-duration tonal segments, spectrally more similar to noise stimuli (both acoustically and perceptually), would not be processed to well-developed frequency representations. Thus perceptual segregation would be less likely to result, and, with respect to gap detection, the stimuli would be more likely to be processed as within-group events.

The perceptual channel theory seems attractive partially because it captures the essence of one's phenomenal impression: the HHLL sequence sounds like two groups of tones. In fact, pilot data on naive Ss indicated that Ss would invariably localize a gap at the greatest frequency juncture in a HGHLL or HHLGL sequence rather than where it actually was. Clearly, the question remains as to whether (a) the frequency disparity produces two channels and hence the perception of temporal groups, or (b) the perceived gap (produced by whatever means-perhaps peripheral) is the cause of such perceptual grouping.

It seems more parsimonious to think that the frequency separation causes a channel separation and hence a perceived gap since the temporal ordering studies discussed previously are unlikely to be explained by a peripheral mechanism. Furthermore, the hypothesis that frequency domains define perceptual channels seems to have a great potential as a unifying concept that can organize the stream segregation, temporal ordering, and gap detection experiments. However, a problem arises if one postulates that the gap detection experiments and the temporal order experiments are explained by the same attention switching mechanism, since the time course appears to be different in the two experiments.

For example, in the present experiments, $80-\mathrm{msec}$ 
gaps are quite detectable (suggesting a switch time of less than $80 \mathrm{msec}$ ), while sequential tracking performance is quite difficult at speeds of $200 \mathrm{msec}$ per tone for widely divergent tones (suggesting a much longer switching time). Two hypotheses could help to explain this discrepancy. First, in the gap detection experiment, the listener has to make only one switch, whereas in the temporal ordering experiments, he has to make repeated switches. It could be that switching time increases in a succession of switches. Furthermore, the breakdown of performance may be due not only to the switching mechanism breaking down after repeated switches, but also to some sort of minimum "dwell time" in each channel (Moray, 1969). Indeed, if the notion of channel is broadened so that any two distinct frequencies can be thought to define separate channels, and if the minimum dwell time is assumed to be greater than $100 \mathrm{msec}$, one could explain the ordering performance of LLLL sequences at rates of 100 /tone. Clearly, the switching time would have to be negligible for tones close in frequency. Thus, the breakdown in performance in a complex frequency tracking task, which underlies a temporal ordering judgment, is probably only partially explainable by the time needed to switch attention, but the relative differences in performance between LLLL and HLHL patterns may, in fact, be due to attention shifting.

\section{REFERENCES}

ABEL, S. M. Discrimination of temporal gaps. Joumal of the Acoustical Society of America, 1972, 52, 519-524.

BÉKÉSY, G. voN. Experiments in hearing. New York: 1960.

Bregman, A. S., \& Campbell, J. Primary auditory stream segregation and perception of order in rapid sequences of tones. Joumal of Experimental Psychology, 1971, 89, 244-249.

Broadbent, D. E. Perception and communication. New York: Pergamon, 1958.
COLlyer, C. E. The detection of a temporal gap between two disparate stimuli. Perception \& Psychophysics, 1974, 16, 96-100.

DAY, R. S. Fusion in dichotic listening. Unpublished doctoral thesis, Stanford University, 1968.

ELFNER, L. F., \& CASKEY, W. E. Continuity effects with alternately sounded noise and tone signals as function of manner of presentation. Joumal of the Acoustical Society of America. $1965,38,543-547$.

Elliot,, L. L. Backward and forward masking of probe tones of different frequencies. Journal of the Acoustical Society of America, 1962, 34, 1116-1117.

Hirsh. I. Auditory perception of temporal order. Journal of the Acoustical Society of America, 1957, 31, 759-767.

Hoмick, J. L., Elfner, L. F., \& Bothe, G. G. Auditory temporal masking and the perception of order. Journal of the Acoustical Society of America, 1969, 45, 712-718.

Moray, N. Attention: Selective processes in vision and hearing. London: Hutchinson Educational, 1969.

Munson, W. A. The growth of auditory sensation. Journal of the Acoustical Society of America, 1947, 19, 584-591.

NeIsSER, U. Cognitive psychology. New York: Appleton-CenturyCrofts, 1967.

Norman, D. A. Temporal confusions and limited capacity processors. Acta Psychologica, 1967, 30, 293-297.

Plomp, R. Rate of decay of auditory sensation. Journal of the Acoustical Society of America, 1964, 36, 277-282.

Thomas, I. B., Hill, P. B., Carroll, F. S., \& Bienvenido, G. Temporal order in the perception of vowels. Journal of the Acoustical Society of America, 1970, 48, 1010-1013.

Thomas, 1. B., \& Fitzgibbons, P. J. Temporal order and perceptual classes. Journal of the Acoustical Society of America, 1971, 50, 86-87. (Abstract)

Treisman, A. M. Pereeption and recall of simultaneous speech stimuli. In A. F. Sanders (Ed.), Attention and performance III. Acta Psychologica, 1970, 33, 132-148.

Treisman, A. M. Shifting attention between the ears. Quarterly Journal of Experimental Psychology, 1971, 23, 157-167.

Warren, R. M., Obusek, C. J., Farmer, R. M., \& Warren, R. P. Auditory sequences: Confusions of patterns other than speech or music. Science, 1969, 164, 586-587.

Williams, K. N., \& Perrott, D. R. Temporal resolution of tonal pulses. Journal of the Acoustical Society of America, 1972, 51, 644-647.

(Received for publication April 26, 1974; revision received July 18,1974 .) 